\title{
Entrainment of sediment particles by very large-scale motions
}

\author{
S. M. Cameron ${ }^{1} \dagger$, V. I. Nikora ${ }^{1}$, and M. J. Witz ${ }^{1}$ \\ ${ }^{1}$ School of Engineering, University of Aberdeen, Aberdeen, AB243UE, United Kingdom
}

(Received xx; revised xx; accepted xx)

Stereoscopic particle image velocimetry (PIV) configured in two orthogonal planes was utilised to capture the flow structure at the instant of entrainment of spherical bed particles in open-channel flow. Experiments were conducted with lightweight target particles amongst a bed of coplanar fixed spheres with diameters of $16 \mathrm{~mm}$. The protrusions of the target particles were set to give an average entrainment rate of $1 / 60 \mathrm{~s}^{-1}$. These protrusions were established from extensive initial experiments which utilised an automated mechanism to place spheres on the bed of the flume and record the time elapsed until they were entrained by the flow. The results showed that at lower flow depth to particle diameter ratios, bed particles are more stable and require larger protrusions to entrain at the same rate as at a larger depth. This effect is consistent with observations of reduced velocity variance and reduced drag force variance for lower flow submergences. The PIV measurements indicated that particle entrainment is associated with very large-scale motions which extend up to 50 flow depths in the streamwise direction. Contributions of smaller scale velocity and pressure spatial fluctuations are suppressed by a spatial averaging effect related to the particle size, and a temporal averaging effect related to the time taken to fully entrain a particle from its resting pocket. These observations are relevant to sediment transport modelling. However, further data are required to clarify the role of particle lift forces, and particle shape in the entrainment process.

Key words:

\section{Introduction}

The interplay between turbulent flows and mobile beds is a classical problem related to a number of practical engineering challenges including: the design of stable channels and structures such as bridge piers; aquatic habitat management; and flood impact assessment (e.g. Graf 1984; Raudkivi 1998; Nikora et al. 2012). Traditional methods of assessing bed stability and transport rates such as Shields' (1936) threshold curve or Einstein's (1950) stochastic approach result in large uncertainties when applied to field conditions. One key constraint to developing refined sediment transport models is that the physical mechanisms involved in the entrainment and motion of sediment particles are not yet well understood at the scale of an individual grain. These mechanisms are the focus of our study. Below we provide some pertinent background information starting with large and very large scale turbulent motions which are likely to induce particle entrainment.

$\dagger$ Email address for correspondence: s.cameron@abdn.ac.uk 


\subsection{Large- and very-large scale motions}

Kim \& Adrian (1999) identified that the pre-multiplied streamwise velocity spectra ( $k S_{u}$, where $k$ is wavenumber and $S_{u}$ is streamwise velocity auto-spectra) in pipe flows had a bi-modal shape and referred to the structures contributing to the respective spectral peaks as large-scale-motions (LSMs) and very-large-scale motions (VLSMs). The bimodal spectral characteristic was subsequently identified also in boundary layer and closed-channel flows (e.g. Hutchins \& Marusic 2007; Monty et al. 2009) and recently in open channel flows (Cameron et al. 2017). In the case of boundary-layer flows, VLSMs are typically referred to as 'superstructures' where they are thought to be confined to the logarithmic flow layer. In other flow types VLSMs can be identified throughout the whole flow domain. Kim \& Adrian (1999) proposed that LSMs identified with streamwise wavelengths of 2-3 times the pipe radius were associated with packets of hairpin shaped vortices and that VLSMs that were found to extend 12-14 pipe radii resulted from the preferential alignment of several hairpin packets. Evidence from boundary-layer (Hutchins \& Marusic 2007) and open-channel (Cameron et al. 2017) flow studies that VLSMs are associated with meandering depth-scale counter-rotating vortical structures, however, suggests a different formation mechanism, possibly associated with a flow instability (e.g. Hwang \& Cossu 2010). Compared to pipe, channel, and boundary layer flows, the VLSMs identified in open channel flow appear to be much longer, often extending up to 50 flow depths in the streamwise direction, although the reasons for the scale difference is yet to be identified. Evidence of the existence of VLSMs in open-channel flows challenges the conventional assumption that the largest turbulent structures are just a few flow depths long (e.g. Nezu \& Nakagawa 1993; Roy et al. 2004; Nezu 2005; Franca \& Brocchini 2015). One reason that the presence of VLSMs in open-channels has been missed until recently is likely due to the fact that their reliable identification requires highresolution and very long-term measurements (several hours for typical laboratory scale conditions), which were not possible previously. Nevertheless, there have been indirect circumstantial indications in a number of earlier studies reflecting the presence of VLSMs in open-channel flows (e.g. Zaitsev 1984; Grinvald \& Nikora 1988; Franca \& Lemmin 2005; Nezu 2005).

\subsection{Origin and scales of drag forces acting on bed particles}

Recent experiments (Cameron et al. 2019) demonstrated that the pre-multiplied frequency spectrum of drag force fluctuations ( $f S_{D}$, where $f$ is frequency and $S_{D}$ is drag force auto-spectra) acting on spherical bed particles has a bimodal shape, with a low frequency peak corresponding to the presence of very-large-scale motions (VLSMs) in the flow, and a higher frequency peak corresponding to the action of turbulent pressure spatial fluctuations (figure 1a). The low frequency peak in figure $1(a)$ is sensitive to the particle protrusion $(P)$ reflecting increased exposure of the particle to the flow. The high frequency peak, in contrast, is much less sensitive to $P$ suggesting that the pressure fluctuations penetrate below the roughness tops exposing the full frontal area of the particle regardless of the protrusion. It is important to distinguish that the spatial pressure fluctuations referred to here are those that exist in the turbulent flow overlying a sediment particle rather than those that can be measured at the particle surface which result from the interaction of the flow field with the particle. The contribution of VLSMs and pressure spatial fluctuations to drag forces on particles was not previously recognised despite a number of studies exploring forces on sediments (e.g. Schmeeckle et al. 2007; Detert et al. 2010; Dwivedi et al. 2011a; Celik et al. 2014). Their contribution should be 
(a)

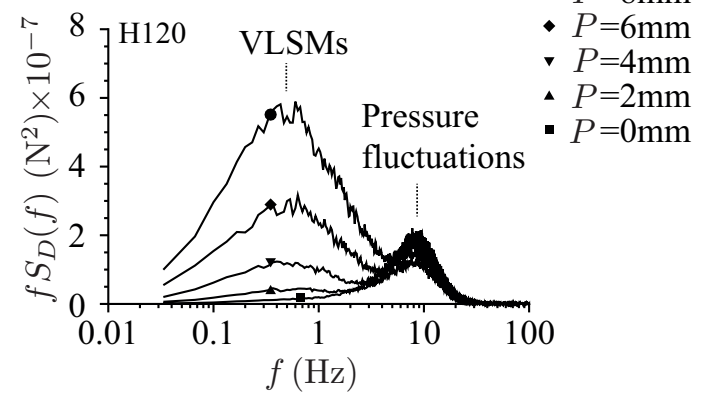

(b)

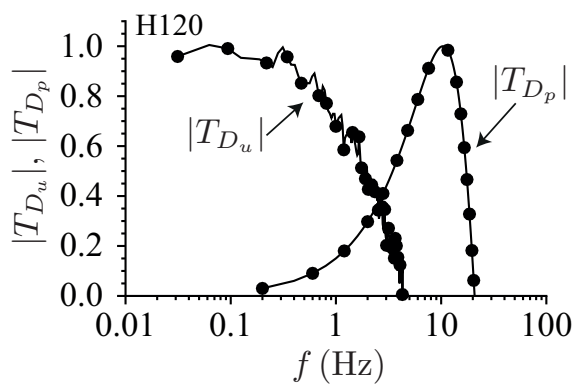

Figure 1. Pre-multiplied spectra of drag force fluctuations for different particle protrusions (a); and gain functions $\left|T_{D_{u}}\right|$ and $\left|T_{D_{p}}\right|(b)$.

incorporated into revised models coupling drag force fluctuations, velocity fluctuations, and pressure field fluctuations.

Assuming quasi-linearity in flow-particle interactions, low external noise, and negligible correlations between the local pressure and velocity fluctuations, it follows from the theory of random functions (e.g. Bendat \& Piersol 2000) that the particle drag force spectra $S_{D}(f)$ can be approximated as a function of the fluid velocity spectra $S_{u}(f)$ and the fluid pressure spectra $S_{p}(f)$ at representative points near the particle as:

$$
S_{D}(f)=\left\{\rho C_{D_{u}} A_{u} \bar{u}\right\}^{2}\left|T_{D_{u}}(f)\right|^{2} S_{u}(f)+\left\{C_{D_{p}} A_{p}\right\}^{2}\left|T_{D_{p}}(f)\right|^{2} S_{p}(f)
$$

where $\rho$ is the fluid density, $C_{D_{u}}$ is a drag-velocity coefficient, $A_{u}$ is exposed frontal area of the particle relevant to velocity fluctuations, $\bar{u}$ is the mean streamwise velocity extracted from a point near the particle, $T_{D_{u}}(f)$ is the dimensionless drag-velocity frequency response function, $C_{D_{p}}$ is a drag-pressure coefficient, $A_{p}$ is the particle frontal area relevant to pressure fluctuations, and $T_{D_{p}}(f)$ is the dimensionless drag-pressure frequency response function. Equation 1.1 combines the leading-order terms contributing to the drag force spectra. In general, additional terms may be required to account for non-Gaussian velocity fluctuations, higher-order relationships between velocity and drag fluctuations (Dwivedi et al. 2010), correlations between pressure and velocity fluctuations (which are typically small due to the non-local property of pressure fluctuations, e.g. Tsinober 2001), and potentially other mechanisms contributing to the drag force. The reference location for the velocity and pressure signals should, in general, be not so close to the particle where the signals are modified by its presence (i.e., it should be outside the particle wake region) but not so far away from the particle for the correlation with the particle drag force to be lost. As a practical measure, Dwivedi et al. (2010) chose a reference point for the velocity field that maximised the correlation coefficient with the particle drag force. Cameron et al. (2019) adopted the same procedure which is also used here. The effective frontal areas $A_{u}$ and $A_{p}$ are not necessarily equivalent and reflect the respective distributions of velocity and pressure around the particle. The gain function $\left|T_{D_{u}}\right|$, i.e. the modulus of the complex valued frequency response function $T_{D_{u}}$, is obtained from the velocity-drag cross-spectrum $S_{u D}$ as:

$$
\left|T_{D_{u}}\right|=\frac{1}{\rho C_{D_{u}} A_{u} \bar{u}} \frac{\left|S_{u D}\right|}{S_{u}}
$$


with

$$
S_{u D}(f)=\frac{1}{T} \int_{0}^{T} u\left(t_{1}\right) e^{i 2 \pi f t_{1}} d t_{1} \int_{0}^{T} F_{D}\left(t_{2}\right) e^{-i 2 \pi f t_{2}} d t_{2}
$$

where $u\left(t_{1}\right)$ is the velocity time series, $F_{D}\left(t_{2}\right)$ is the drag force time series, $T$ is the time span, and $i$ is the imaginary unit. The function $T_{D_{u}}$ reflects the averaging of small-scale velocity fluctuations over the spatial domain with volume comparable to the particle volume and is illustrated in figure 1(b) from the data presented in Cameron et al. (2019). The drag-pressure gain function $\left|T_{D_{p}}\right|$ is defined using the pressure-drag cross-spectrum $S_{p D}$ as:

$$
\left|T_{D_{p}}\right|=\frac{1}{C_{D_{p}} A_{p}} \frac{\left|S_{p D}\right|}{S_{p}}
$$

with

$$
S_{p D}(f)=\frac{1}{T} \int_{0}^{T} p\left(t_{1}\right) e^{i 2 \pi f t_{1}} d t_{1} \int_{0}^{T} F_{D}\left(t_{2}\right) e^{-i 2 \pi f t_{2}} d t_{2}
$$

where $p\left(t_{1}\right)$ is the pressure time series. The function $T_{D_{p}}$ acts as a differencing filter reflecting that the drag force is proportional to the pressure difference between upstream and downstream particle faces. Data are not available yet to directly estimate $\left|T_{D_{p}}\right|$. Cameron et al. (2019), however, suggest that it is reasonably approximated by $\left|T_{D_{p}}\right| \approx \sin \left(\pi f D / u_{c}\right)$ which is plotted in figure $1(b)$, where $u_{c}$ is the convection velocity of pressure fluctuations. Together, the gain functions $T_{D_{u}}$ and $T_{D_{p}}$ (figure 1) define the time scales of velocity and pressure fluctuations, respectively, that contribute to particle drag force and potentially entrainment.

Equation 1.1 can also be obtained by considering a time-domain parameterisation for the instantaneous drag force as:

$$
F_{D}(t)=0.5 \rho C_{D_{u}} A_{u}[\dot{u}(t)]^{2}+C_{D_{p}} A_{p} \Delta_{p}(t)
$$

and following a derivation procedure similar to that used in Naudascher \& Rockwell (1994) and (Dwivedi et al. 2010), where $\dot{u}(t)$ is the streamwise component of velocity near the particle after filtering to remove high frequency fluctuations that do not contribute to the drag force, and $\Delta_{p}(t)$ is the pressure difference in the flow above the particle at a streamwise separation equal to the particle diameter. Similar filtering of the streamwise velocity component has previously been proposed by Nelson et al. (1995) after identifying that low frequency velocity fluctuations were contributing a majority of the sediment transport. Such parametrisation of the drag force may be implemented in sediment transport models (e.g. Schmeeckle \& Nelson 2003; Ancey et al. 2008; Ali \& Dey 2016) to more accurately account for the scales of velocity fluctuations contributing to drag forces and incorporate the role of pressure spatial fluctuations. Insufficient data, however, are currently available to generalise the behaviour of $A_{u}, A_{p}, C_{D_{p}}, C_{D_{u}}, T_{D_{u}}(f), T_{D_{p}}(f)$ and the pressure and velocity spectra $\left(S_{p}(f)\right.$ and $S_{u}(f)$ respectively) over a range of flowsubmergences $(H / D$ where $H$ is flow depth and $D$ is particle diameter), particle Reynolds numbers $\left(D^{+}=D u_{*} / \nu\right.$ where $u_{*}$ is shear velocity and $\nu$ is fluid kinematic viscosity), particle relative protrusions $(P / D)$ and particle shapes.

Fluctuating lift forces on particles have proven more difficult to analyse than drag forces with Schmeeckle et al. (2007) and Dwivedi et al. (2011b) reporting poor correlation with the local streamwise or vertical fluid velocity. Hofland \& Booij (2004) on the other hand found a relation between the vertical velocity component and lift, but this result 
is likely uniquely related to their flat-topped particle with a single pressure sensor to approximate the lift force. Considering spatial fluctuations in the pressure field rather than the velocity field, Smart \& Habersack (2007) proposed that lift forces generated by pressure fluctuations often exceeded particle weight forces and could cause particle entrainment. This role of spatial pressure fluctuations suggests that a modified version of equation 1.1 may also be applicable to parameterise particle lift forces.

Overall, the indications of figures $1(a)$ and $1(b)$ and the analysis of Cameron et al. (2019) are that as VLSMs contribute significantly to particle drag forces, they should also directly contribute to particle entrainment, particularly at larger protrusions. This hypothesis will be tested in this study using particle image velocimetry (PIV) recordings of the flow field leading up to, during, and after the instant of the entrainment of single spherical particles.

\subsection{Objectives}

The first objective of the study is to explore the relationship between drag force fluctuations and particle entrainment which is a key component of sediment transport models (e.g. Einstein 1950; Ancey et al. 2008). While it is straightforward to define a threshold entrainment condition where drag and lift forces are balanced by the particle weight and friction with the bed, it is known that the destabilising forces need to persist for sufficient duration to completely displace a particle from its resting pocket (e.g. Diplas et al. 2008; Celik et al. 2010; Valyrakis et al. 2010; Maldonado \& de Almeida 2019). The cited authors identify the force impulse, i.e. the product of force and duration, as the key parameter characterising particle entrainment. Their studies, however, largely relate to the conditions of maximum particle protrusion, with single spherical particles overlying a co-planar spherical particle bed. We will, in this study, explore the relationship between drag force fluctuations and entrainment at low and intermediate particle protrusions $(P<0.5 D)$. To do this we will compare mean waiting-times until entrainment estimated from drag force time series with those obtained from single particle entrainment experiments. The waiting-time is defined as the elapsed time before a resting particle is entrained by the flow. For independent entrainment events, the waiting-time is expected to follow an exponential distribution (e.g. Cinlar 2013) characterised by a single parameter, the entrainment rate $\lambda_{t}$ where $\lambda_{t}^{-1}$ is the mean waiting-time. For a given flow condition, the mean waiting-time is a function of the particle protrusion with increasing $P$ expected to correspond to decreasing $\lambda_{t}^{-1}$. We can define the protrusion corresponding to a particular mean waiting-time as $P_{\lambda^{-1}}\left(D^{+}, \rho_{s} / \rho, H / D\right)$, where $\rho_{s}$ is the particle density and $\rho$ is the fluid density. In this study we will establish and utilise $P_{\lambda^{-1}}=P_{60}$, i.e. the protrusion corresponding to a mean waiting-time of 60 seconds, by recording waiting times for single particles over a range of $\rho_{s} / \rho$ and $H / D$ with constant $D^{+}$. This first objective provides new information regarding interrelations between fluctuating drag and entrainment events and also underpins the PIV entrainment experiments.

The second objective of this study is to explore the relationship between the velocity field and particle entrainment events. To do this we used stereoscopic particle image velocimetry to record the velocity fields during entrainment events over a range of $\rho_{s} / \rho$ and $H / D$. These experiments were conducted with particle protrusions that resulted in a standardised 60 second mean waiting time with $P=P_{60}$ which is the outcome of the first objective. Similar experiments have been conducted in the past focussing on single particles to identify 'coherent structures' responsible for entrainment (e.g. Hofland \& Booij 2004; Dwivedi et al. 2011a; Wu \& Shih 2012) along with more general studies of mobile beds (Sutherland 1967; Séchet \& Le Guennec 1999). No convincing evidence has emerged that there is a dominant 'coherent structure' responsible for entrainment except 
for the general observation that entrainment is correlated with 'sweep' events (i.e. with the streamwise velocity fluctuation greater than zero, and the vertical velocity fluctuation negative) which might be associated with Adrian's (2007) type hairpin vortices. Hofland \& Booij (2004) identified that 'sweep' events allowed the flow to penetrate deeper into the bed increasing drag forces on a cube shaped particle, while, at the same time, producing negative lift forces due the downward directed flow. Similarly, Sutherland (1967) hypothesised eddies that disrupted the viscous sublayer and directly impinged on the particle surface to be responsible for entrainment. Séchet \& Le Guennec (1999) in contrast claimed a more significant contribution of low speed 'ejection' events. These studies, however, pre-date the observations of VLSMs in OCFs (Cameron et al. 2017), and it is timely to re-investigate this matter with specially targeted experiments, i.e. with multiple measurement plane orientations and with extended fields of view.

The structure of the paper is as follows. In $\S 2$ we describe the flow conditions and equipment used for two types of experiments: firstly to establish the mean waiting-time until entrainment across different flow depths and particle densities; and secondly to reveal the velocity field at the time of entrainment. In $\S 3$ we present our experimental results and in the final section we summarise our main findings.

\section{Experimental setup}

Experiments were conducted in the Aberdeen Open-Channel Facility (AOCF) using the same flow and bed conditions as in Cameron et al. (2017) and Cameron et al. (2019). The bed was made of a single layer of $16 \mathrm{~mm}$ diameter $(D)$ glass spheres in a hexagonally close-packed arrangement. The flow depth $(H)$ varied between $30 \mathrm{~mm}$ and $120 \mathrm{~mm}$ (table 1 ) while adjusting the bed slope $\left(S_{0}\right)$ to keep the shear velocity $u_{*}=\sqrt{g H S_{0}}$ constant, where $g$ is acceleration due to gravity. The roughness Reynolds number $D^{+}=D u_{*} / \nu$ was 605 indicating fully-rough bed conditions. The flows were steady, uniform, and the large flow width to depth ratio $(B / H>10)$ ensured that the central region of the flow away from the sidewalls was fairly two-dimensional and generally free of secondary currents (Cameron et al. 2017). Target experiments for this study were conducted using flow conditions H030, H070, and H120 (table 1). The H050 and H095 flow parameters are retained in table 1 as we will re-use in our analysis some of the data from Cameron et al. (2017) and Cameron et al. (2019).

For this study we have performed two types of experiments: (1) waiting-time experiments to address the first objective (§1.3), and (2) synchronous stereoscopic particle image velocimetry (PIV) with entrainment of a single mobile particle to achieve the second objective. These are described below.

\subsection{Waiting-time experiments}

In order to measure the distribution of waiting-times until entrainment of individual particles, we constructed a computer-controlled device to automatically place a sphere onto the bed of the flume, record the time until it was entrained by the flow, and then load a new sphere. The time of entrainment of the target particle was determined by a fibrecoupled photo diode beneath the target sphere which indicated increased light intensity when the sphere was not present. The optical fibre was mounted inside a $1 \mathrm{~mm}$ diameter vertically-orientated steel tube which could be height adjusted to control the protrusion of the particle between $P=0$ (co-planar) and $P=0.5 D$. For a given flow condition, the mean waiting-time is expected to decrease with increasing particle protrusion. We chose a target mean waiting-time of $60 \mathrm{~s}$ and performed experiments to establish the particle protrusion corresponding to this mean waiting-time (i.e. $P_{60}$ ). The $60 \mathrm{~s}$ period is somewhat arbitrary, 


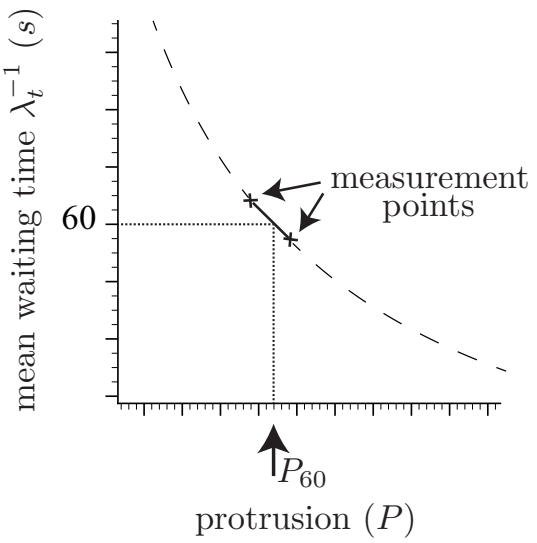

FIgURE 2. Illustration of how the protrusion $P_{60}$ corresponding to a mean waiting time between entrainments of $60 \mathrm{~s}$ is obtained.

but it needed to be long enough that it was possible to place the particle in a stable position on the bed, and short enough to allow a sufficient number of entrainment events to be captured.

Experiments were performed with spheres made of Nylon (' $\mathrm{N}$ ') with a density of 1.12 $\mathrm{g} / \mathrm{cm}^{3}$ and Delrin ('D') with a density of $1.38 \mathrm{~g} / \mathrm{cm}^{3}$. We recorded the waiting-times for 500 entrainment events with a protrusion resulting in a mean waiting-time of between $40 \mathrm{~s}$ and $60 \mathrm{~s}$ and 500 events with a protrusion resulting in a mean waiting-time between $60 \mathrm{~s}$ and $80 \mathrm{~s}$. The protrusion for $60 \mathrm{~s}$ mean waiting-time $\left(P_{60}\right)$ was then calculated by linear interpolation of the mean waiting-time versus $P$ curve (e.g. figure 2 ). Uncertainty in the estimation of $P_{60}$ using this method was approximately $\pm 0.1 \mathrm{~mm}$. This procedure was repeated for ' $\mathrm{N}$ ' and ' $\mathrm{D}$ ' spheres with flow conditions H030, H070, and H120 (table $1)$.

\subsection{Particle image velocimetry with a single mobile particle}

To assess the flow structure at the instant of particle entrainment we have used stereoscopic PIV in two planes, 'cross-flow' and 'streamwise' (figure $3 a$ ). The 'cross-flow' plane extended $320 \mathrm{~mm}$ in the transverse direction and was centred at the mid-point of the flume cross-section. The 'streamwise' plane extended $340 \mathrm{~mm}$ upstream of the target particle and $200 \mathrm{~mm}$ downstream, i.e. covering a total streamwise extent of $540 \mathrm{~mm}$. Both configurations covered the flow region from the roughness tops to the water surface. The 'cross-flow' plane PIV configuration is equivalent to that reported in detail in Cameron et al. (2017) and Cameron et al. (2019). To setup the 'streamwise' plane we have reorientated the light sheet to enter the water around $1 \mathrm{~m}$ downstream of the measurement area via an immersed $20 \mathrm{~mm}$ prism. The four cameras used for the 'cross-flow' plane were split into two groups of two cameras, with one group covering the upstream $270 \mathrm{~mm}$ and the other group covering the downstream $270 \mathrm{~mm}$ with a small overlap between the measuring regions of each group. The PIV processing algorithms and parameters were the same as those used in Cameron et al. (2017) and Cameron et al. (2019). For the 'streamwise' plane, the two subregions were combined in post-processing to create a seamless $540 \mathrm{~mm}$ wide measuring region.

We used the 'cross-flow' and 'streamwise' configurations to record 25 entrainment events for each of the ' $\mathrm{N}$ ' and ' $\mathrm{D}$ ' particles at their respective $P_{60}$ protrusion with flow conditions H030, H070, and H120. In total we recorded 150 entrainment events with the 'cross-flow' configuration and 150 entrainment events with the 'streamwise' 
(a)

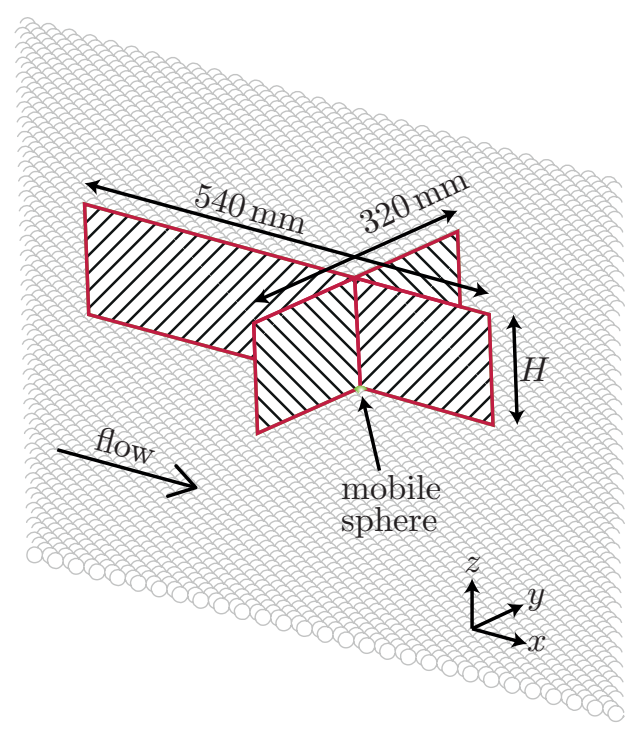

(b)

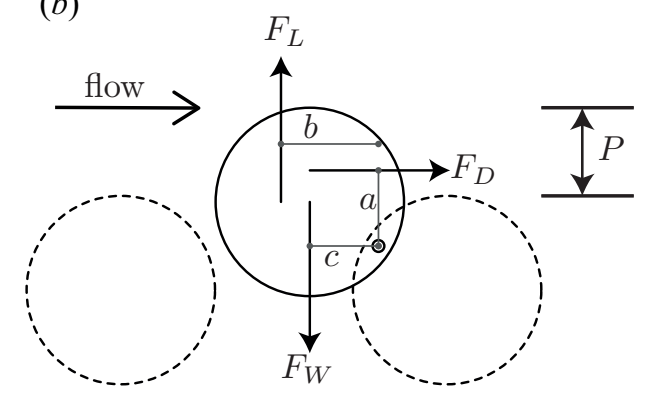

(c)

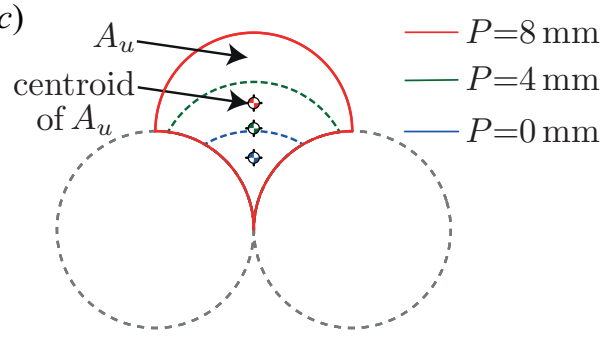

FiguRE 3. Transverse and streamwise PIV measurement planes relative to mobile sphere $(a)$; forces and force lever arms acting on a sphere $(b)$; and frontal area and centre of area for different particle protrusions $(c)$.

configuration. The recording duration covered the $30 \mathrm{~s}$ prior to the entrainment time and $5 \mathrm{~s}$ afterwards. The sampling frequency was $100 \mathrm{~Hz}, 50 \mathrm{~Hz}$, and $32 \mathrm{~Hz}$ for $\mathrm{H} 030, \mathrm{H} 070$, and H120, respectively. Additionally, we used the 'streamwise' configuration with a fixed coplanar bed and a recording duration of 10 minutes to measure directly the wavenumber velocity spectra for the H030, H070, and H120 flows. This data are reported in $§ 3.1$.

\section{Results}

\subsection{Background flow statistics}

As reported in Cameron et al. (2017), the double-averaged (in time and in space) streamwise velocity $\langle\bar{u}\rangle$ for the studied flow conditions exhibits a logarithmic scaling range for elevations $0.5 D<z<0.5 H$, despite the small relative submergence $(H / D)$. The 

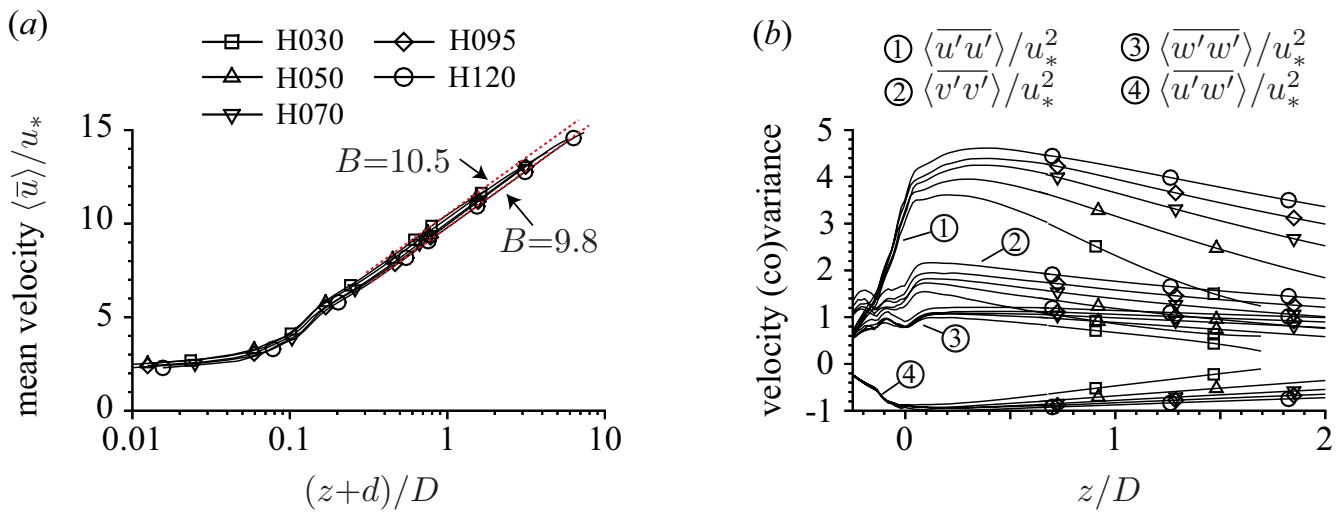

Figure 4 . Mean velocity $(a)$ and velocity variance $(b)$. The roughness tops are at $z=0$ while the dashed lines in $(a)$ are the $\log$ law with $\kappa=0.38, d=1.7 \mathrm{~mm}$ and offset $B$ as indicated, $u^{\prime}$, $v^{\prime}$, and $w^{\prime}$ are streamwise, transverse, and vertical velocity fluctuations, respectively. Angular brackets define spatial averaging and overbar defines time averaging.

von Kàrmàn constant $\kappa$ was found to be 0.38 with a zero-plane displacement $d \approx 1.7 \mathrm{~mm}$, i.e., the 'virtual bed' is just below the roughness tops which are at $z=0$. Both the von Kàrmàn constant and the zero-plane displacement appeared to be only very weakly dependent on the relative submergence. Figure $4(a)$ shows that the additive term $B$ in the logarithmic law

$$
\frac{\langle\bar{u}\rangle}{u_{*}}=\frac{1}{\kappa} \ln \left(\frac{z+d}{D}\right)+B
$$

increases from $B=9.8$ for $\mathrm{H} 120$ to $B=10.5$ for $\mathrm{H} 030$ as the relative submergence $H / D$ decreases from 7.5 to 1.9 (table 1 ). Above $0.5 H$, the velocity distributions deviate only slightly from the log law and are pseudo-logarithmic through most of the flow depth. Towards the bed, the velocity gradient increases and reaches a maximum near the roughness tops.

Second-order statistics (figure $4 b$ ) reveal a clear effect of decreasing streamwise velocity variance with decreasing relative submergence. We demonstrated in Cameron et al. (2019) that below the roughness tops the velocity variances tend to collapse as a function of $z / D$ whereas in the outer flow the profiles converge if expressed as a function of $z / H$. Just above the roughness tops neither scaling holds and the velocity variances are a function of $H / D$. Higher-order statistics, two-point correlation functions and pre-multiplied spectra for these flow conditions are reported in Cameron et al. (2017).

The 'streamwise' plane PIV measurements described in $\S 2.2$ permit estimates of velocity spectra directly in the wave-number domain, compared to the approximation of applying Taylor's hypothesis to frequency domain measurements in Cameron et al. (2017). Therefore it is worth re-examining velocity spectra with this new data, particularly given its relationship to the drag force spectra (i.e. equation 1.1). The PIV window size is not sufficiently large to directly resolve VLSMs. However, the directly-measured wavenumber spectra extend to higher wavenumbers $(k=2 \pi / \lambda$, where $\lambda$ is wavelength) compared to the frequency domain based estimates. Figures 5 and 6 therefore report hybrid spectra, via frequency domain using Cameron et al.'s (2017) data for $k<50 \mathrm{~m}^{-1}$ and direct wavenumber spectra estimates using newly collected data for $k>50 \mathrm{~m}^{-1}$.

Near-bed streamwise velocity spectra $S_{u}$ are expected to collapse across two ranges of the normalised wavenumbers $(k z)$ with $S_{u} \propto(k z)^{-1}$ for the '-1' scale range and $S_{u} \propto(k z)^{-5 / 3}$ for inertial subrange scales (e.g. Perry et al. 1987; Raupach et al. 1991; 

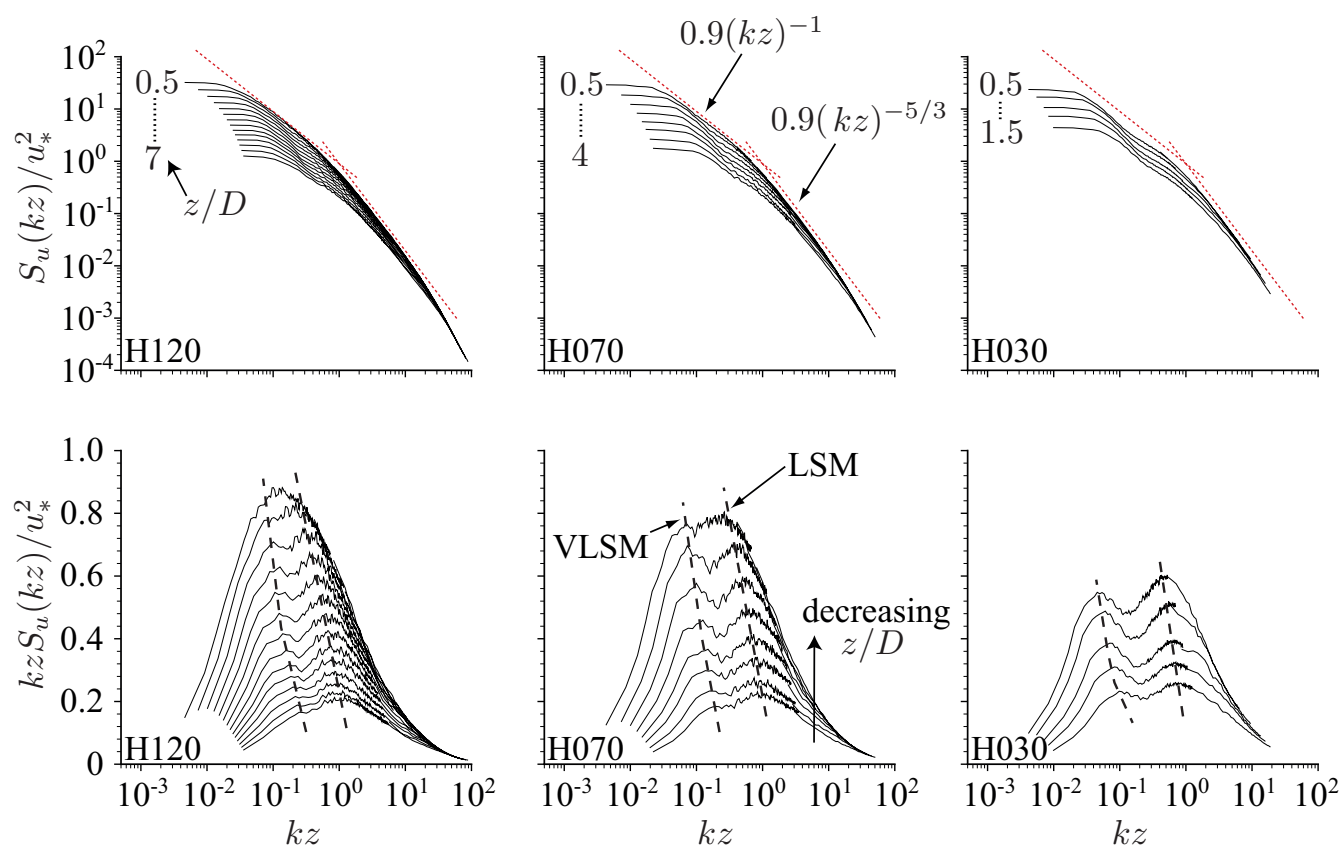

FiguRE 5. Auto-spectra (top row) and pre-multiplied auto-spectra (bottom row) of streamwise velocity fluctuations at different elevations. Red dashed lines are the scaling ranges of Nikora \& Goring (2000).
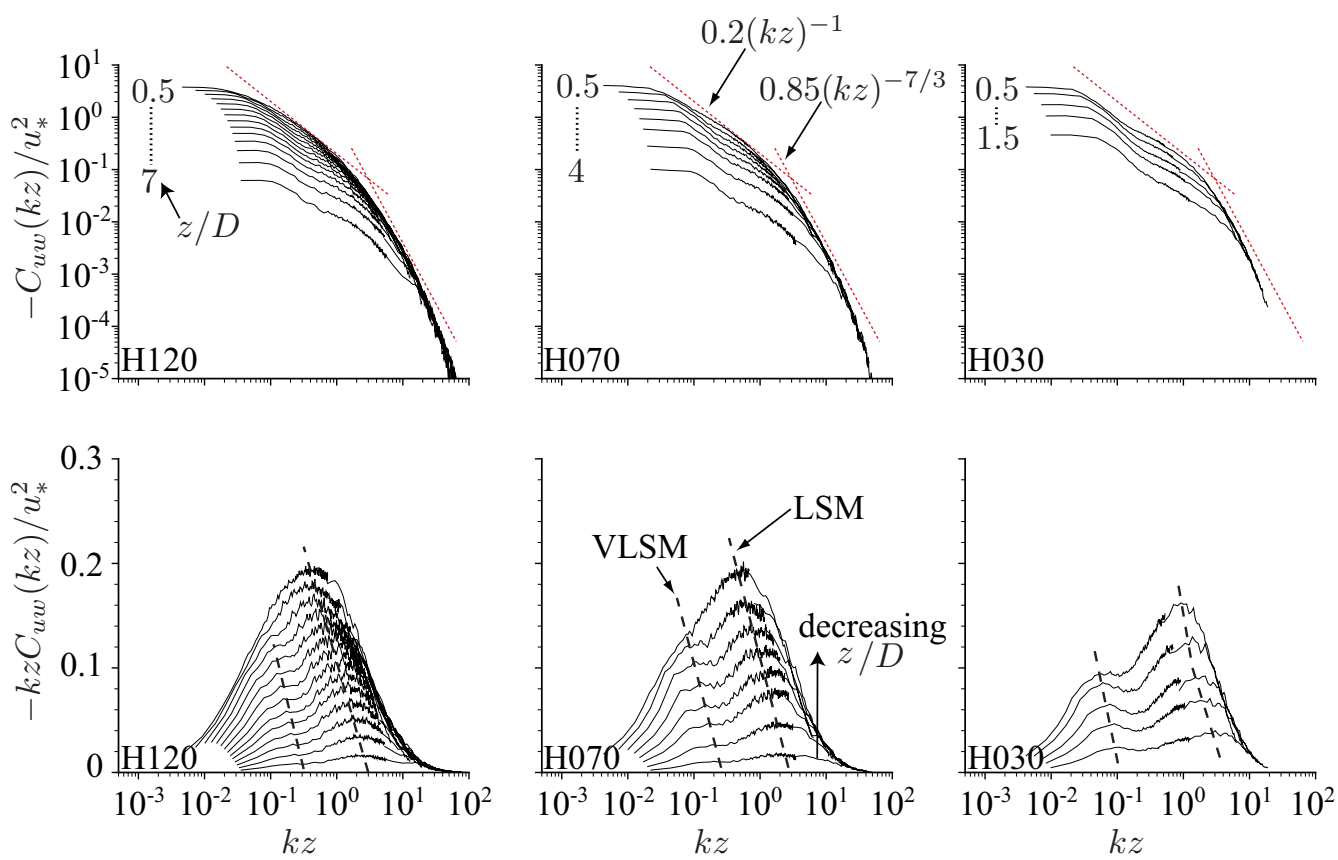

Figure 6. Co-spectra (top row) and pre-multiplied co-spectra (bottom row) of streamwise-vertical velocity fluctuations at different elevations. Red dashed lines are the scaling ranges of Nikora \& Goring (2000). 
Nikora \& Goring 2000). Similarly, the co-spectra $-C_{u w}$ are expected to exhibit analogous ranges where $-C_{u w} \propto(k z)^{-1}$ and $-C_{u w} \propto(k z)^{-7 / 3}$. Figures 5 and 6 suggest that for the studied flows the Reynolds number is not high enough to support an extended inertial subrange, while the small relative submergence restricts the extent of the '-1' range. Nevertheless, for H120 our data approach the $\propto k z^{-1}$ trend reported in Nikora \& Goring (2000) for high Reynolds number field experiments $(R=200000-780000)$ which is marked by dashed lines in figures 5 and 6 . For H070 and H030 the measured spectra and co-spectra drop below the Nikora \& Goring (2000) trend in the '-1' range consistent with the submergence effect identified for the streamwise velocity variance. The kink in the spectra at low wavenumbers due to VLSMs becomes clearer with decreasing submergence and the pre-multiplied spectra $k z S_{u}(k z)$ reveal the expected bi-modal shape. It is interesting to note in the H120 case that near-bed '-1' scaling appears to co-exist with VLSMs in the higher flow layers. This corresponds to the apparent bifurcation in spectra $k S_{u}(k) / u_{*}=f(\lambda / H, z / H)$ reported in Cameron et al. (2017) and also seen in figure 5 where the pre-multiplied spectra transitions from having a single peak near the bed to a bi-modal shape at larger elevations. For all flows, the measured spectra are somewhat below the Nikora \& Goring (2000) trend for the inertial range in high-Re open channel flow. This may result from the lower Reynolds number of our laboratory experiments. It is interesting to note from the pre-multiplied spectra (figure 5 and 6) that VLSMs contribute substantially (approaching 40\%) to the streamwise velocity variance, but slightly less to the Reynolds stress (approximately 30\%). Below $0.5 \mathrm{D}$ the velocity variance is spatially heterogeneous and dominated by wake regions behind individual roughness elements (e.g. Cameron et al. 2019). Velocity spectra in the range of $z<0.5 D$ are therefore highly dependent on the roughness geometry, and it is unlikely that any universal scaling of the spectra for this range of elevations can be defined.

\subsection{Mean waiting-time}

The protrusion corresponding to a mean waiting-time until entrainment of $60 \mathrm{~s}$ (i.e. $\left.P_{60}\right)$ is plotted against the flow depth for the Nylon ' $\mathrm{N}$ ' and Delrin ' $\mathrm{D}$ ' spheres in figure 7 (circle and square symbols, respectively). As described in $\S 2.1, P_{60}$ for each configuration was estimated based on 1000 timed entrainment events. The ' $\mathrm{N}$ ' spheres were found to entrain with a protrusion of $\approx 2 \mathrm{~mm}$ while the higher density ' $\mathrm{D}$ ' spheres required protrusions of $6-7 \mathrm{~mm}$. For both sphere materials, particles have higher stability at lower submergences and thus require larger protrusions to entrain at the same rate as at larger depths. This is consistent with the observation that the near-bed streamwise velocity variance and the drag force variance decrease as the flow depth is reduced (Cameron et al. 2019).

The $P_{60}$ versus $H$ curve can also be estimated using the 90 minute duration drag force time series for fixed particles from Cameron et al. (2019) which cover the parameter space $P=0-8 \mathrm{~mm}$ and $H=30-120 \mathrm{~mm}$. To do this, we solve the moment balance equation for near horizontal beds $a F_{D c}+b F_{L c}-c F_{W}=0$ (figure $3 b$ ) for $F_{D c}$ and count the number of independent events in the time series with recorded force greater than $F_{D c}$, subject to a minimum event duration $t_{c}$ (figure $7 b$ ). Here $F_{D c}$ is the critical drag force on the particle, $F_{L c}$ is the critical lift force, $F_{W}=g\left(\rho_{s}-\rho\right) \pi D^{3} / 6$ is the immersed weight force, $a$ is the drag force lever arm, $b$ is the lift force lever, and $c$ is the weight lever. Lift force measurements are not available for these conditions so we set $F_{L c}=0$. The lever arms $a$ and $c$ were calculated such that $F_{D c}$ and $F_{W}$ passed through the frontal area centroid (figure $3 c$ ) and the volumetric centre of the particle, respectively. The result of this procedure is the surface of mean waiting-time in the plane $(P, H)$ for a given $t_{c}$ and 


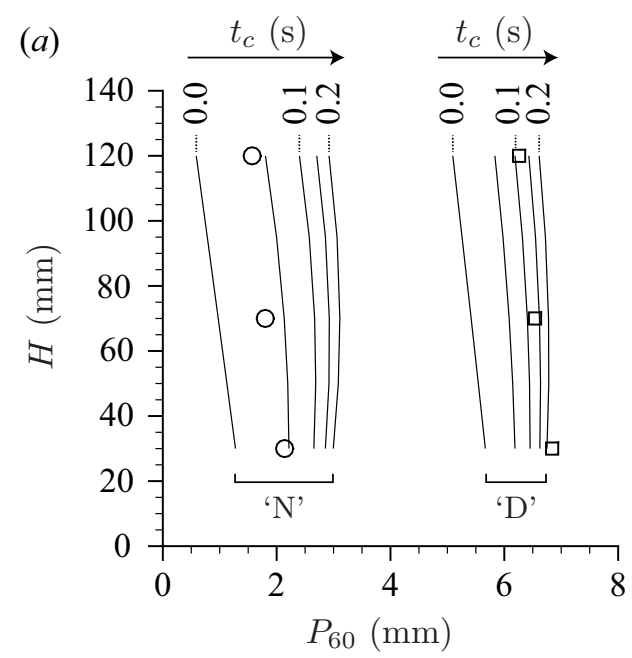

(b)

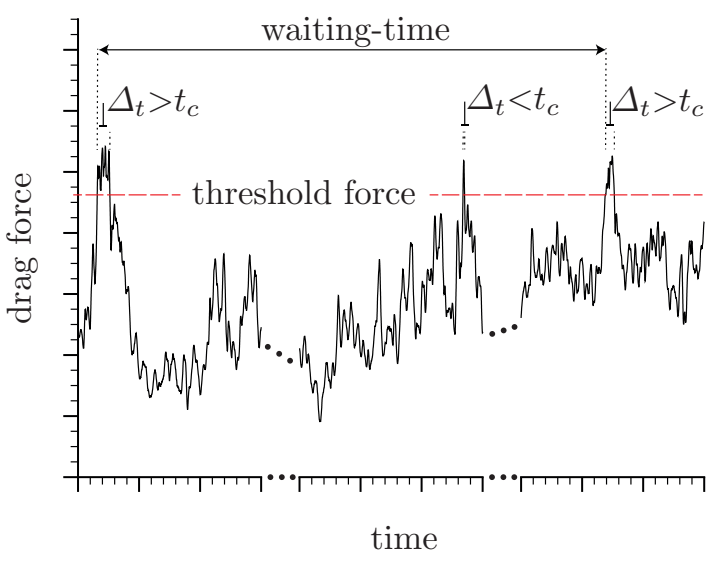

Figure 7. ( $a$ ) Protrusion $\left(P_{60}\right)$ corresponding to an entrainment rate of $1 / 60 \mathrm{~s}$ for Nylon ' $\mathrm{N}$ ' and Delrin ' $\mathrm{D}$ ' spheres for different flow depths (symbols). Solid lines in $(a)$ are $P_{60}$ inferred from drag force time series where entrainment events are defined as shown in $(b)$ by the drag force exceeding a threshold force for a duration $\left(\Delta_{t}\right)$ exceeding a critical duration $\left(t_{c}\right)$. Drag force time series data were taken from Cameron et al. (2019).

$\rho_{s}$. It is then straight-forward to extract the contour of $60 \mathrm{~s}$ mean waiting-time which is shown in figure 7 . We have chosen to use a minimum event duration threshold $t_{c}$ in this analysis instead of a minimum force impulse threshold (e.g. Celik et al. 2010) because physical values of $t_{c}$ are easier to interpret in the context of turbulence scales, i.e., figures 1,5 , and 6 .

It is immediately clear that for a minimum event duration threshold equal to zero (i.e. $\left.t_{c}=0\right), P_{60}$ is underestimated compared to the single particle entrainment data, even without considering potential contributions from the lift force. For ' $\mathrm{N}$ ' spheres, the single particle entrainment data correspond to $t_{c}$ of approximately $0.05 \mathrm{~s}$, while for ' $\mathrm{D}$ ' spheres the required event duration is around $0.1-0.2 \mathrm{~s}$. It seems reasonable that the ' $\mathrm{N}$ ' spheres entrain with shorter event durations, as due to their lower density they can accelerate faster in response to an unbalanced force and therefore fully entrain in a shorter time. Although figure 7 indicates that the critical event duration $t_{c}$ increases with decreasing submergence, i.e from $0.1 \mathrm{~s}$ for H120 to $0.2 \mathrm{~s}$ for H030 with ' $\mathrm{D}$ ' spheres, it is not clear why. It may be the result of submergence effects on turbulence scales and energy (figure 5 ) or the potential role of the lift force which was neglected in this analysis.

\subsection{Ensemble average flow field}

Ensemble average velocity fluctuation fields at the time of particle entrainment were estimated as

$$
\widehat{u_{i}^{\prime}}=\frac{1}{N} \sum^{N} u_{i_{n}}\left(x, y, z, t=t_{n}\right)-\bar{u}(x, y, z)
$$

where $u_{i_{n}}(x, y, z, t)$ is the velocity field for the $n$th repeated experiment, $t_{n}$ is the time corresponding to the start of particle motion in the $n$th ensemble and $N=25$ is the number of repeated experiments for each flow condition and particle protrusion. Averaging in this way preserves flow features that are common across repeated entrainment events while suppressing random deviations from the common pattern. It is important to note that the ensemble average of velocity fluctuation fields sampled at random times (i.e. 

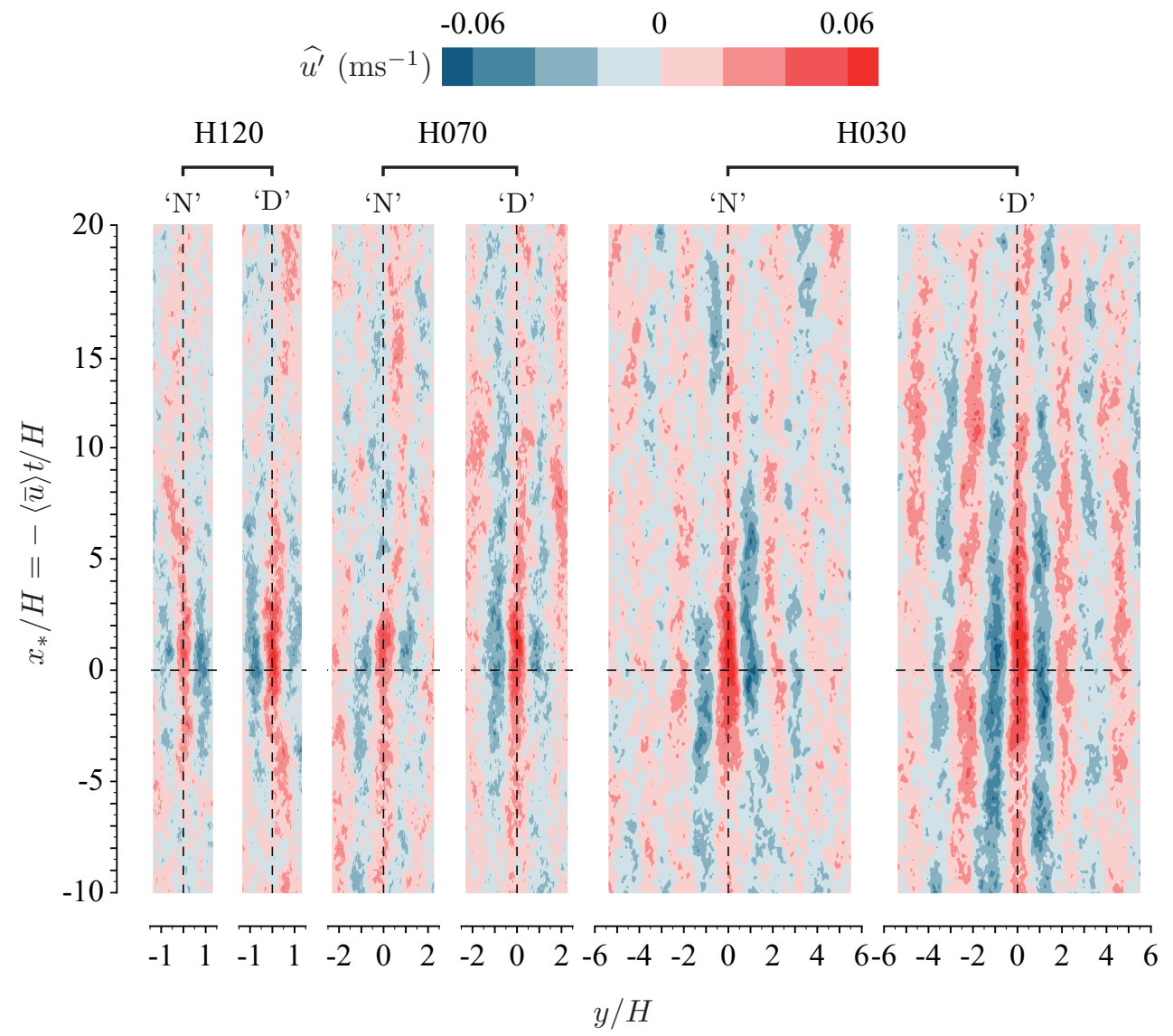

FiguRE 8. Ensemble average of streamwise velocity fluctuation at $z / H=0.5$ at time of entrainment. Mobile particle is at $x_{*} / H=0, y / H=0$.

replacing $t_{n}$ in equation 3.2 with a random time coordinate) converges to zero. Therefore, non-zero values of $\widehat{u}_{i}^{\prime}$ can be interpreted as the flow structures associated with (or causing) particle entrainment. Such ensemble averaged flow fields are reported in figures 8 to 10 for the $i=1$ streamwise component. The 'cross-flow' (figure 9) and 'streamwise' (figure 10) planes were recorded directly, however, the bed-parallel plane (figure 8) is a reconstruction from velocity time series before and after entrainment using a convection velocity equal to $\langle\bar{u}\rangle(z)$. The ensemble average fields were calculated from 25 recorded entrainment events at the $P_{60}$ protrusion for each flow condition and particle density. Due to the relatively small number of events contributing to the ensemble average, some patchiness is evident in the $\widehat{u^{\prime}}$ contours. Nevertheless, the elongated streaks of alternating high and low momentum fluid with $2 H$ transverse period (figure 8) clearly indicate that VLSMs are the key contributor to the ensemble average. Compared to the instantaneous velocity fluctuation fields reported in Cameron et al. (2017), the $\widehat{u^{\prime}}$ fields are smoother and the meandering characteristic of the VLSMs is suppressed due to the ensemble averaging. The alternating streaks for the high protrusion Delrin ('D') particles appear to be better defined compared to the low protrusion Nylon ('N') cases. This effect may reflect the observation that VLSMs contribute less to the particle drag force (and therefore entrainment) as the protrusion is reduced (figure 1a) and the higher frequency 
a)

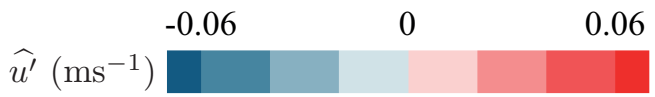

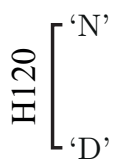

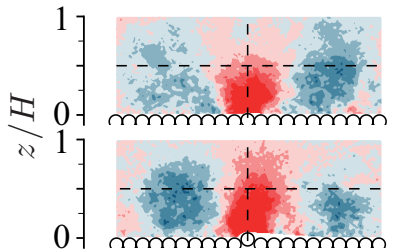

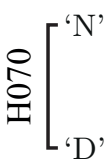
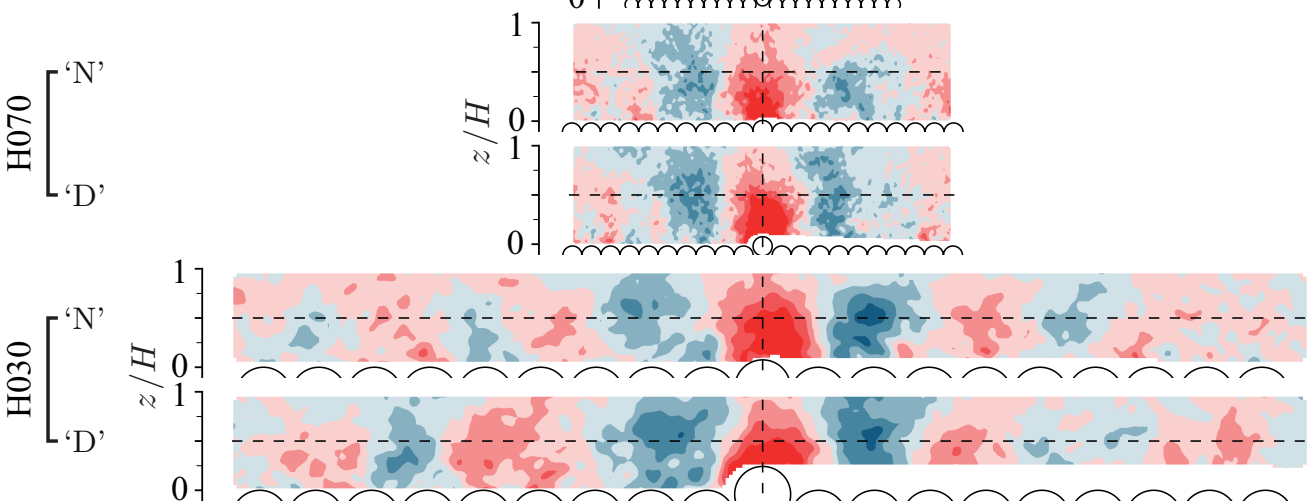

b)

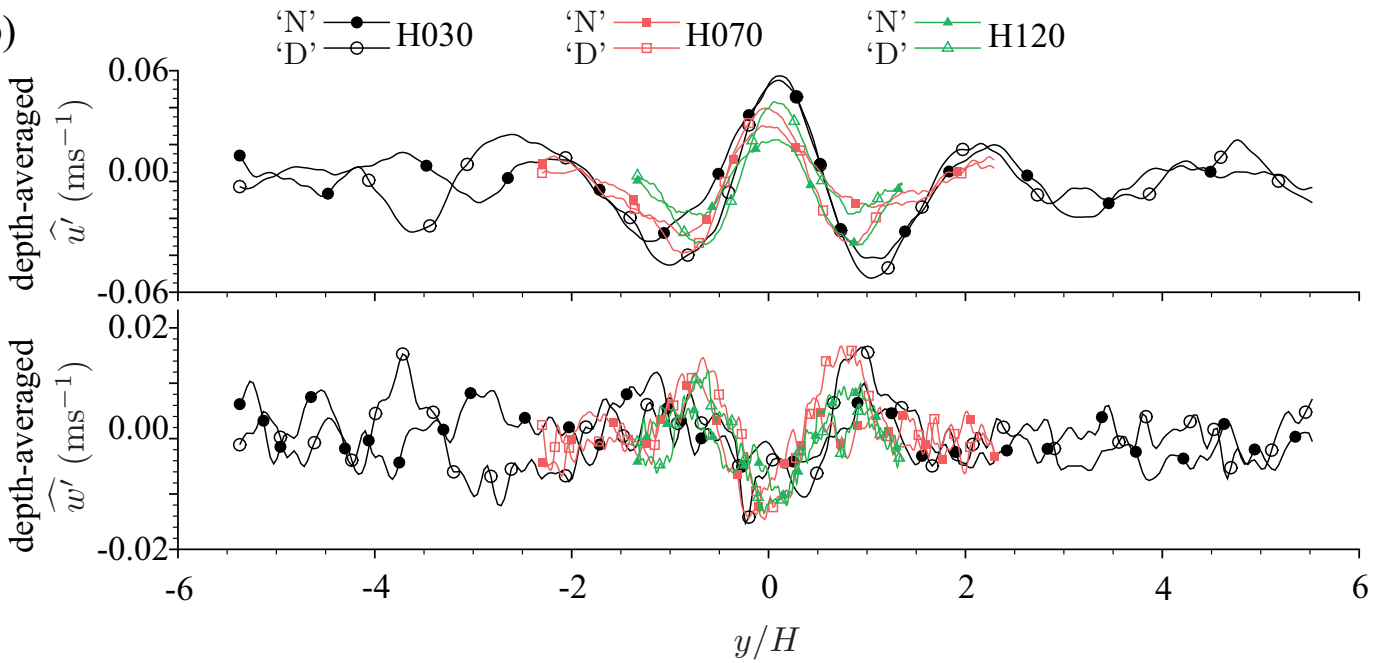

Figure 9. Ensemble average of streamwise velocity fluctuation in the transverse plane at time of entrainment (a), and depth averaged velocity fluctuation at time of entrainment (b).

pressure fluctuations due to the passage of smaller scale structures become relatively more important (Cameron et al. 2019).

Figure 9(a) indicates that the VLSMs occupy near the entire flow depth from the roughness tops to the water surface such that the transverse periodicity of the velocity fluctuation is preserved after depth averaging (figure 9b). Figure 9b indicates that the transverse wavelength of the fluctuations is close to $2 \mathrm{H}$ for $\mathrm{H} 030$, but narrows slightly as the flow depth is increased to H120. A similar shortening of the transverse wavelength of 

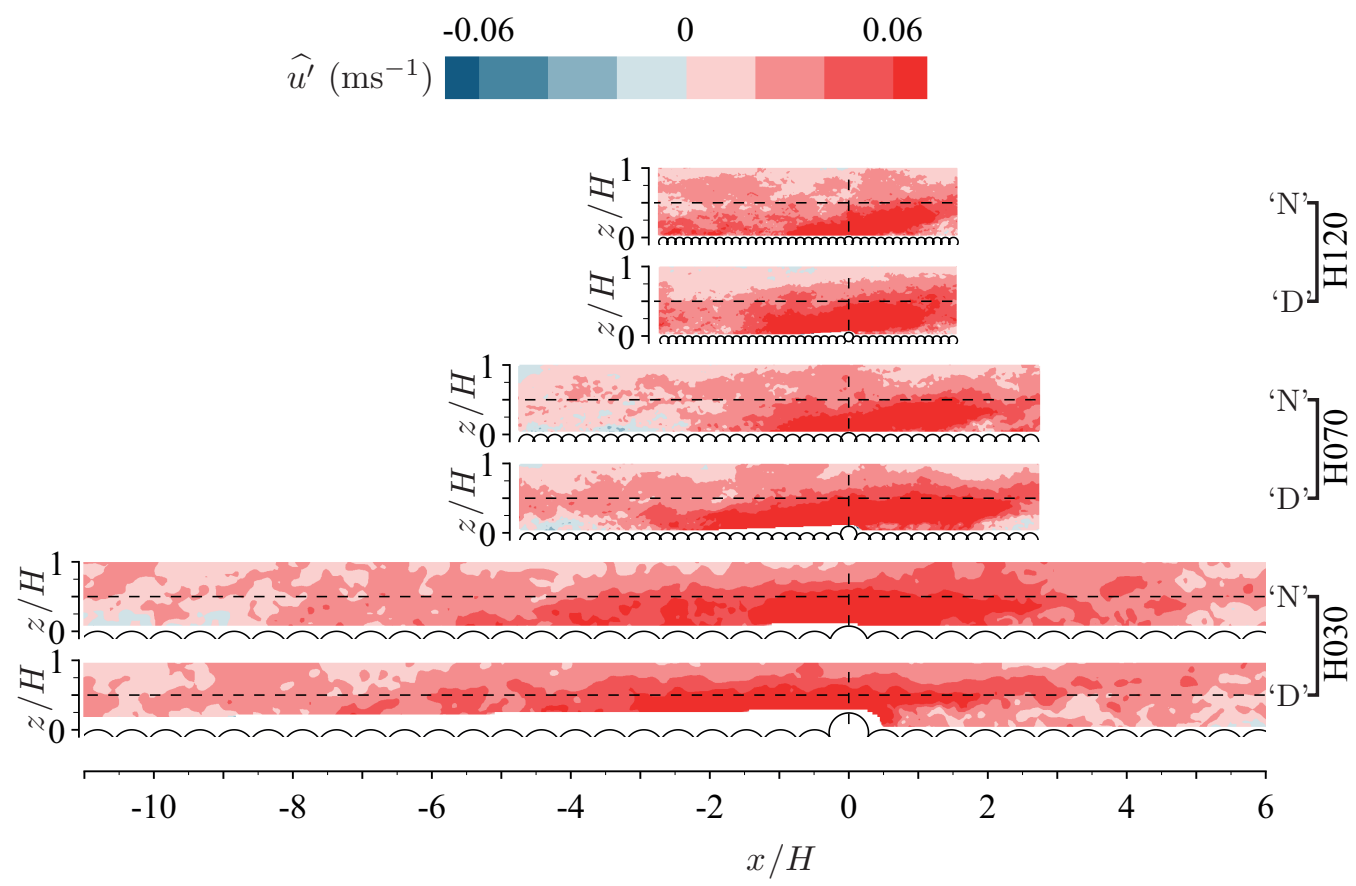

Figure 10. Ensemble average of streamwise velocity fluctuation in the streamwise plane at time of entrainment. Flow is from left to right.

VLSMs with increasing relative submergence was also noted in Cameron et al. (2017), but the origin of the effect is not yet known. Figure 9(b) also shows the depth average of the ensemble averaged vertical velocity fluctuation. Although the vertical velocity component is quite small and therefore not as well resolved in the ensemble average as the streamwise velocity component, a clear downflow region aligned with particle is seen, with upflow regions to the sides aligned with the zones of low streamwise momentum. This result is consistent with the depth-scale counter-rotating vortical structure of VLSMs (e.g. Hutchins \& Marusic 2007; Cameron et al. 2017).

Figure 10 shows that the $\widehat{u^{\prime}}$ contours are inclined with respect to the bed. This inclination likely results from the mean shear stretching the flow features as they evolve. At the instant of entrainment the target particle is immersed in the high velocity region of the VLSM where the drag force is maximised. For H030 the VLSMs appear longer in terms of flow depths compared to H070 and H120 consistent with the scaling noted in Cameron et al. (2017).

The role of VLSMs in the particle entrainment process identified in figures 8 to 10 is consistent with previous indications (Cameron et al. 2019) that they contribute significantly to drag force fluctuations. In general, we can identify two reasons why very large scale structures are favoured. Firstly, the contribution of small-scale velocity fluctuations to the drag force are suppressed by averaging over the spatial domain with volume comparable to the particle volume. This is described by the gain function $\left|T_{D_{u}}\right|$ (equation 1.1, figure $1 b$ ). Secondly, the minimum force event duration $\left(t_{c}\right)$ to completely entrain a particle acts as an additional filter, suppressing the contribution of higher frequency drag force fluctuations. For example, with a $t_{c}$ of $0.1-0.2 \mathrm{~s}$ for ' $D$ ' particles (figure 7 ), the $\approx 10 \mathrm{~Hz}$ drag force fluctuations (figure $1 a$ ) that relate to pressure spatial fluctuations in the overlying turbulent flow, likely contribute very little to particle 
entrainment. For the ' $\mathrm{N}$ ' spheres, however, with a $t_{c}$ of $\approx 0.05 \mathrm{~s}$, and reduced sensitivity of the drag force to VLSMs at the lower protrusion (figure $1 a$ ), the $\approx 10 \mathrm{~Hz}$ pressure spatial fluctuations may play a more important role. Further data are required, with direct measurements of turbulent pressure fluctuations to confirm their contribution to particle entrainment.

\subsection{Instantaneous flow field}

In addition to the ensemble average velocity fluctuation fields, we have explored the instantaneous fields for each of the 300 recorded entrainment events for evidence of smaller scale 'coherent structures' contributing to entrainment. At the studied Reynolds numbers, however, the instantaneous fields appear as a random collection of vortices with different scales and orientations. It appears unlikely that any particular structure of analytical value relevant to sediment transport could be extracted. This, however, might be reviewed when high resolution volumetric data become available.

\section{Conclusions}

The ensemble average of velocity fields corresponding to the instant of particle entrainment demonstrate that sediment transport is strongly linked to VLSMs in the flow. In particular, entrainment of single spherical particles occurs when the high momentum region of a VLSM overlays a particle. Pressure spatial fluctuations which lead to a $\approx 10 \mathrm{~Hz}$ peak in pre-multiplied drag force spectra may also contribute to particle entrainment. This is particularly true for particles with small protrusion which have reduced exposure to the VLSMs. The contribution of small-scale velocity fluctuations is suppressed by a spatial averaging effect associated with the particle size. Furthermore, drag and lift force fluctuations need to persist for sufficient duration to completely entrain a particle from its resting cavity. This minimum event duration limits the contribution of high frequency force fluctuations to the entrainment process. A relative submergence effect is seen in entrainment rate data which indicates that particle stability increases with decreasing flow depth under constant shear velocity conditions. This effect is also seen in the drag force variance and likely relates to suppression of the large scale turbulence due to the limited separation between flow depth and roughness length scales. Further data are required to extend these observations to a wider range of relative flow submergence and particle Reynolds number, and to ascertain the potential role of particle lift forces which is still unclear.

\section{Acknowledgements}

The study has been supported by two EPSRC/UK grants, "High-resolution numerical and experimental studies of turbulence-induced sediment erosion and near-bed transport" (EP/G056404/1) and "Bed friction in rough-bed free-surface flows: a theoretical framework, roughness regimes, and quantification" (EP/K041088/1). The authors are grateful to three anonymous reviewers and the Editor for constructive criticisms and helpful suggestions that improved presentation of the material in the paper.

\section{Declaration of Interests}

The authors report no conflict of interest. 


\section{REFERENCES}

Adrian, R. J. 2007 Hairpin vortex organization in wall turbulence. Phys. Fluids 19 (4), 041301. Ali, Sk Z. \& Dey, S. 2016 Hydrodynamics of sediment threshold. Phys. Fluids 28 (7), 075103. Ancey, C., Davison, A. C., Böhm, T., Jodeau, M. \& Frey, P. 2008 Entrainment and motion of coarse particles in a shallow water stream down a steep slope. J. Fluid Mech. 595, 83-114.

Bendat, J.S. \& Piersol, A.G. 2000 Random data analysis and measurement procedures. IOP Publishing.

Cameron, S. M., Nikora, V. I. \& Marusic, I. 2019 Drag forces on a bed particle in openchannel flow: Effects of pressure spatial fluctuations and very-large-scale motions. J. Fluid Mech. 863, 494-512.

Cameron, S. M., Nikora, V. I. \& Stewart, M. T. 2017 Very-large-scale motions in roughbed open-channel flow. J. Fluid Mech. 814, 416-429.

Celik, A.O., Diplas, P., Dancey, C.L. \& Valyrakis, M. 2010 Impulse and particle dislodgement under turbulent flow conditions. Phys. Fluids 22 (4), 046601.

Celik, A. O., Diplas, P. \& Dancey, C. L. 2014 Instantaneous pressure measurements on a spherical grain under threshold flow conditions. J. Fluid Mech. 741, 60-97.

Cinlar, ERHAN 2013 Introduction to stochastic processes. Courier Corporation.

Detert, M., Nikora, V. \& Jirka, G. H. 2010 Synoptic velocity and pressure fields at the water-sediment interface of streambeds. J. Fluid Mech. 660, 55-86.

Diplas, P., Dancey, C.L., Celik, A.O., Valyrakis, M., Greer, K. \& Akar, T. 2008 The role of impulse on the initiation of particle movement under turbulent flow conditions. Science 322 (5902), 717-720.

Dwivedi, A., Melville, B.W. \& Shamseldin, A.Y.And Guha, T.K. 2011a Flow structures and hydrodynamic force during sediment entrainment. Water Resour. Res. 47 (1).

Dwivedi, A., Melville, B. W., Shamseldin, A. Y. \& Guha, T. K. 2010 Drag force on a sediment particle from point velocity measurements: A spectral approach. Water Resour. Res. 46 (10).

Dwivedi, A.J, Melville, B. W., Shamseldin, A. Y. \& Guha, T. K. $2011 b$ Analysis of hydrodynamic lift on a bed sediment particle. J. Geophys. Res.-Earth 116 (F2).

Einstein, H. A. 1950 The bed-load function for sediment transportation in open channel flows. US Department of Agriculture Washington, DC.

Franca, M. J. \& Brocchini, M. 2015 Turbulence in rivers. In Rivers-Physical, Fluvial and Environmental Processes, pp. 51-78. Springer.

FrancA, M. J. \& Lemmin, U. 2005 Cross-section periodicity of turbulent gravel-bed river flows. Proc. of the 4th River, Coastal and Estuarine Morphodynamics: RCEM 2005 1, 203-210.

Graf, W. H. 1984 Hydraulics of sediment transport. Water Resources Publication.

Grinvald, D. I. \& Nikora, V. I. 1988 River turbulence. Hydrometeoizdat, Leningrad, Russia.

Hofland, B. \& Boois, R. 2004 Measuring the flow structures that initiate stone movement. River Flow 2004 pp. 821-830.

Hutchins, N. \& MARusic, I. 2007 Evidence of very long meandering features in the logarithmic region of turbulent boundary layers. J. Fluid Mech. 579, 1-28.

Hwang, Y. \& Cossu, C. 2010 Self-sustained process at large scales in turbulent channel flow. Phys. Rev. Lett. 105 (4), 044505.

Kim, K. C. \& Adrian, R. J. 1999 Very large-scale motion in the outer layer. Phys. Fluids $11(2), 417-422$.

Maldonado, S. \& DE Almeida, G.A.M. 2019 Theoretical impulse threshold for particle dislodgement. J. Fluid Mech. 863, 893-903.

Monty, J. P., Hutchins, N., NG, H. C. H., Marusic, I. \& Chong, M. S. 2009 A comparison of turbulent pipe, channel and boundary layer flows. J. Fluid Mech. 632, 431-442.

Naudascher, E. \& Rockwell, D. 1994 Flow-Induced Vibrations: An Engineering Guide. Dover.

Nelson, J. M., Shreve, R. L., Mclean, S. R. \& Drake, T. G. 1995 Role of near-bed turbulence structure in bed load transport and bed form mechanics. Water resour. res. 31 (8), 2071-2086. 
Nezu, I. 2005 Open-channel flow turbulence and its research prospect in the 21st century. $J$. Hydraul. Eng. 131 (4), 229-246.

Nezu, I. \& Nakagawa, H. 1993 Turbulence in Open Channel Flows. Balkema.

Nikora, V., Cameron, S., Albayrak, I., Miler, O., Nikora, N., Siniscalchi, F., Stewart, M. \& O'Hare, M. 2012 Flow-biota interactions in aquatic systems: Scales, mechanisms, and challenges. In Environmental Fluid Mecahanics: Memorial Volume in Honour of Prof. Gerhard H. Jirka (ed. W. Rodi \& M. Uhlmannm), chap. 11, pp. 217-235. CRC Press.

Nikora, V. \& Goring, D. 2000 Flow turbulence over fixed and weakly mobile gravel beds. $J$. Hydraul. Eng. 126 (9), 679-690.

Perry, A.E., Lim, K.L. \& Henbest, S.M. 1987 An experimental study of the turbulence structure in smooth-and rough-wall boundary layers. J. Fluid Mech. 177, 437-466.

RaudKivi, Arved J 1998 Loose boundary hydraulics. CRC Press.

Raupach, M.R., Antonia, R.A. \& Rajagopalan, S. 1991 Rough-wall turbulent boundary layers. Appl. mech. rev. 44 (1), 1-25.

Roy, A. G., Buffin-Belanger, T., Lamarre, H. \& Kirkbride, A. D. 2004 Size, shape and dynamics of large-scale turbulent flow structures in a gravel-bed river. J. Fluid Mech. 500, $1-27$.

SchmeECKle, M. W. \& Nelson, J. M. 2003 Direct numerical simulation of bedload transport using a local, dynamic boundary condition. Sedimentology 50 (2), 279-301.

Schmeeckle, M. W., Nelson, J. M. \& Shreve, R. L. 2007 Forces on stationary particles in near-bed turbulent flows. J. Geophys. Res.-Earth 112 (F2).

SÉchet, P. \& Le Guennec, B. 1999 Bursting phenomenon and incipient motion of solid particles in bed-load transport. J. Hydraul. Res. 37 (5), 683-696.

SHIELDS, A. 1936 Anwendung der aehnlichkeitsmechanik und der turbulenzforschung auf die geschiebebewegung. PhD thesis, Technical University Berlin.

Smart, G. M. \& Habersack, H. M. 2007 Pressure fluctuations and gravel entrainment in rivers. J. Hydraul. Res. 45 (5), 661-673.

Sutherland, A. J. 1967 Proposed mechanism for sediment entrainment by turbulent flows. $J$. Geophys. Res. 72 (24), 6183-6194.

Tsinober, A. 2001 An informal introduction to turbulence. Springer Science \& Business Media.

Valyrakis, M., Diplas, P., Dancey, C.L., Greer, K. \& Celik, A.O. 2010 Role of instantaneous force magnitude and duration on particle entrainment. J. Geophys. Res.Earth 115 (F2).

Wu, F.C. \& SHIH, W.R. 2012 Entrainment of sediment particles by retrograde vortices: Test of hypothesis using near-particle observations. J. Geophys. Res.-Earth 117 (F3).

Zaitsev, N.I. 1984 Large-scale eddy structure of turbulent flow in a straight channel. Proc. of the State Hydrological Institure, St Petersburg, Russia 318, 3-17. 